\title{
Heme-arginate suppresses phospholipase $C$ and oxidative stress in the mesenteric arterioles of mineralcorticoid-induced hypertensive rats
}

\author{
Joseph Fomusi Ndisang and Ashok Jadhav
}

Induction of heme-oxygenase (HO) is an important cellular defense mechanism against oxidative and inflammatory insults. We analyzed the effects of the $\mathrm{HO}$ inducer, heme-arginate, on the phospholipase C (PLC)/inositol-triphosphate (IP ( $_{3}$ ) pathway in the mesenteric arterioles of uninephrectomized (UnX) deoxycorticosterone acetate (DOCA)-salt hypertensive rats, which is a volumeoverload model characterized by elevated endothelin (ET-1) and mineralocorticoid-induced oxidative/inflammatory insults. Our study included the following groups: (A) controls [(i) surgery-free Sprague-Dawley (SD) rats, (ii) UnX-Sham, (iii) UnX-Salt (0.9\% $\mathrm{NaCl}+0.2 \% \mathrm{KCl}$ ) and (iv) UnX-DOCA)]; (B) UnX-DOCA-salt hypertensive rats; (C) UnX-DOCA-salt+heme-arginate; (D) UnX-DOCAsalt+heme-arginate+chromium mesoporphyrin (CrMP), the HO inhibitor; (E) UnX-DOCA-salt+CrMP (F); SD+heme-arginate, (G) UnX-DOCA-salt+vehicle dissolving heme-arginate and CrMP and (H) normal-SD+heme-arginate. Quantitative reverse transcriptase PCR, western blot, enzyme immunoassay and spectrophotometric analyses were used. Heme-arginate enhanced mesenteric arteriole HO-1, HO activity, cyclic guanosine monophosphate (cGMP) and anti-oxidants including bilirubin, ferritin, superoxide dismutase with potentiation of the total anti-oxidant capacity. Correspondingly, oxidative/inflammatory mediators such as 8-isoprostane, nuclear-factor $к B$ (NF-кB) and ET-1 were markedly reduced. Furthermore, heme-arginate suppressed PLC activity, attenuated $\mathrm{IP}_{3}$ and reduced resting intracellular calcium. The effects of heme-arginate were nullified by the $\mathrm{HO}$ inhibitor, with aggravation of oxidative/inflammatory insults. In heme-arginate-treated SD rats, the HO system was potentiated to a lesser magnitude and the suppression of ET-1, PLC, IP 3 and NF-кB were less accentuated, suggesting greater selectivity of HO against the ET-1-PLC-IP $3-$ NF-кB destructive axis in the pathological condition of mineralocorticoid-induced hypertension. Given

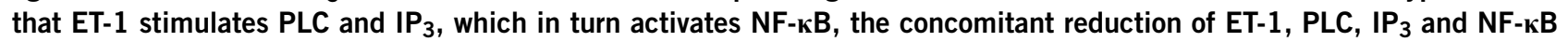
alongside the corresponding decline of resting intracellular calcium may account for the reduction of blood pressure and attenuation of oxidative/inflammatory injury by heme-arginate.

Hypertension Research (2010) 33, 338-347; doi:10.1038/hr.2010.1; published online 5 March 2010

Keywords: DOCA-salt hypertension; endothelin; heme-oxygenase; mesenteric arterioles; NF- $\kappa \mathrm{B}$

\section{INTRODUCTION}

Uninephrectomized (UnX) deoxycorticosterone acetate (DOCA)-salt hypertension is a model of human primary aldosteronism that is characterized by elevated endothelin-1 (ET-1) and high levels of the synthetic mineralcorticoid (DOCA) with severe tissue injury. ${ }^{1}$ Although aldosterone is traditionally known as mineralcorticoid that promotes sodium/water retention, recent evidence indicates that it triggers the formation of superoxide, which in turn quenches nitric oxide by forming peroxynitrite, ${ }^{2}$ which subsequently oxidizes arachidonic acid, generating 8-isoprostane, a potent vasoconstrictor and stimulator of ET- $1 .{ }^{3}$ Similarly, ET-1 causes oxidative injury besides its anti-diuretic/sodium-retention responses. ${ }^{4}$ Moreover, aldosterone and ET-1 stimulate each other reciprocally. ${ }^{5-7}$ Thus, the elevated levels of mineralcorticoid and 8-isoprostane in DOCA hypertension constitute a powerful vasoconstrictor axis that amplifies the vasopressor action of
ET-1..$^{2,7-10}$ Therefore, strategies capable of suppressing ET-1/aldosterone-induced insults would be useful to restore tissue function.

Phospholipase C (PLC) catalyzes the breakdown of phosphoinositides to inositol 1,4,5-triphosphate $\left(\mathrm{IP}_{3}\right)$ and diacylglycerol. ${ }^{11}$ The $\mathrm{PLC}-\mathrm{IP}_{3}$ second messenger system mobilizes intracellular calcium and potentiates vascular contractility with subsequent elevation of blood pressure. ${ }^{12,13}$ Moreover, in DOCA hypertension, the high levels of the synthetic mineralcorticoid would further potentiate vascular contractility through stimulation of PLC and diacylglycerol. ${ }^{12,13}$ As UnXDOCA hypertension is characterized by elevated ET-1, and ET-1 stimulates PLC and $\mathrm{IP}_{3},{ }^{14}$ which in turn activates nuclear-factor $\kappa \mathrm{B}$ $(\mathrm{NF}-\kappa \mathrm{B}),{ }^{15,16} \mathrm{ET}-1, \mathrm{PLC}, \mathrm{IP}_{3}, 8$-isoprostane and NF- $\kappa \mathrm{B}$ may constitute a potent oxidative/inflammatory destructive axis. Interestingly, NF- $\kappa \mathrm{B}$ activates heme-oxygenase (HO-1) by binding to the promoter region of HO-1 gene. ${ }^{17,18} \mathrm{HO}$ is a microsomal enzyme with inducible 
(HO-1) and constitutive (HO-2) isoforms. ${ }^{19}$ The pharmacological upregulation of the $\mathrm{HO}$ system has been shown to combat hypertension, inflammation and oxidative stress. ${ }^{20-26}$ By breaking down the heme moiety, HO removes the pro-oxidant heme ${ }^{21}$ while carbon monoxide, bilirubin/biliverdin and ferritin scavenge reactive oxygen species, inhibit lipid peroxidation and suppress tissue inflammation..$^{22-26}$ Accordingly, carbon monoxide, bilirubin, bliverdin and ferritin constitute a protective tetrad against hypertension and its secondary damages including oxidative and inflammatory insults.

Although upregulating the $\mathrm{HO}$ system is cytoprotective, ${ }^{20-26}$ the effects of the $\mathrm{HO}$ inducer, heme-arginate, on ET-1, PLC, $\mathrm{IP}_{3}$, 8 -isoprostane and NF- $\kappa \mathrm{B}$ in the mesenteric arterioles of mineralcorticoid-induced hypertension have not been reported. Therefore, this study will delineate the cytoprotective role of heme-arginate in second-order branches of the mesenteric arteries, given the importance of resistance vasculature in BP regulation.

\section{METHODS}

\section{Animals, treatment groups and biochemical parameters}

Our experimental protocol was approved by the committee on animal care and research ethics of the University of Saskatchewan, which is in conformity with the Guide for Care and Use of Laboratory Animals stipulated by the Canadian Council on Animal Care and the National Institutes of Health (NIH Publication no. 85-23, revised 1996). Male Sprague-Dawley (SD) rats of 8 weeks of age were purchased from Charles River Laboratories (Willington, MA, USA). The animals were housed at $21{ }^{\circ} \mathrm{C}$ with 12 -h light/dark cycles, fed with standard laboratory chow and had access to drinking water ad libitum. After a week of acclimatization, DOCA-salt hypertension was induced in some animals as previously described. ${ }^{27-29}$

Our experimental protocol included the following groups ( $n=6-10$ per group): (A) controls [(i) surgery-free or normal SD rats; (ii) SD rats with one kidney removed or UnX-sham, (iii) UnX rats that received water containing $0.9 \% \mathrm{NaCl}+0.2 \% \mathrm{KCl}$ (UnX-salt) and (iv) UnX rats implanted s.c. at the midscapular region with a silastic strip impregnated with DOCA (100 mg per kg body weight; Sigma-Aldrich, St Louis, MO, USA) (UnX-DOCA)]; (B) UnX rats implanted with DOCA strip that received water containing $0.9 \%$ $\mathrm{NaCl}+0.2 \% \mathrm{KCl}$ (UnX-DOCA-salt hypertensive rats); (C) UnX-DOCA-salt rats treated with (D) the $\mathrm{HO}$ inducer, heme-arginate or (E) heme-arginate together with the HO blocker, chromium mesoporphyrin (CrMP), or (F) with CrMP alone, or $(\mathrm{G})$ the vehicle dissolving heme-arginate and CrMP. In addition, the final group was $(\mathrm{H})$ normal SD rats treated with heme-arginate.

The involvement of the $\mathrm{HO}$ system in the heme-arginate-induced effects was ascertained by giving the HO blocker, CrMP $\left(4 \mu \mathrm{mol} \mathrm{kg}{ }^{-1}\right.$ i.p., Porphyrin Products, Logan, UT, USA), with or without heme-arginate $\left(30 \mathrm{mg} \mathrm{kg}^{-1}\right.$, i.p.). ${ }^{30}$ Heme-arginate and CrMP were dissolved in $0.1 \mathrm{M} \mathrm{NaOH}$, titrated to $\mathrm{pH} 7.4$ with $0.1 \mathrm{M} \mathrm{HCl}$ and diluted 1:10 with phosphate buffer as we have previously reported. ${ }^{27,28,31,32}$ Although many $\mathrm{HO}$ inhibitors are nonspecific and may affect other hemo-enzymes or even increase HO-1, we had previously shown that using CrMP at a dose of $4 \mu \mathrm{mol} \mathrm{kg}^{-1}$ is selective against HO. ${ }^{30}$

Treatment with heme-arginate, CrMP, or the vehicle began after the animals were severely hypertensive with a blood pressure of $197.8 \pm 4.6 \mathrm{~mm} \mathrm{Hg}$. Each injection was of $0.5 \mathrm{ml}$, and was given twice weekly for 4 weeks. Systolic blood pressure was determined weekly in conscious animals by standard tail-cuff noninvasive method (Model 29-SSP, Harvard Apparatus, St Laurent, Quebec, Canada) during therapy. At the end of the 4 -week treatment period, the study was terminated. The animals were weighed, anesthetized with pentobarbital sodium (50 $\mathrm{mg} \mathrm{kg}^{-1}$ i.p.) and killed, and blood samples were collected and second-order branches of the mesenteric arteries were isolated and harvested.

\section{Isolation of total RNA and quantitative reverse transcriptase-PCR for $\mathbf{p} 65 \mathrm{NF}-\mathrm{kB}$}

This assay was carried out as we previously reported. ${ }^{27,28}$ Samples of mesenteric arterioles were homogenized in $0.5 \mathrm{ml}$ Trizol Reagent (Invitrogen Life Technologies, Carlsbad, CA, USA) according to the manufacturer's specifications.
Reverse transcription was carried out using First-Strand cDNA Synthesis Kit (Novagen, Madison, WI, USA) with $0.5 \mu \mathrm{g}$ Oligo (dT)6, $50 \mathrm{~mm}$ Tris- $\mathrm{HCl}(\mathrm{pH}$ 8.3 at $25^{\circ} \mathrm{C}$ ), $75 \mathrm{~mm} \mathrm{KCl}, 3 \mathrm{~mm} \mathrm{MgCl} 2,50 \mathrm{~mm}$ dithiothreitol, $10 \mathrm{~mm}$ each free deoxynucleotide triphosphate and $100 \mathrm{U}$ of MMLV (Moloney Murine leukemia virus) reverse transcriptase according to the manufacture's specifications. Quantitative PCR was performed using Applied Biosystems 7300 Real Time PCR system (Foster City, CA, USA) and iQ SYBR Green Supermix (Bio-Rad, Hercules, CA, USA) containing $50 \mathrm{~mm} \mathrm{KCl,} 20 \mathrm{~mm}$ Tris- $\mathrm{HCl}$ (pH 8.4), $0.2 \mathrm{~mm}$ each free deoxynucleotide triphosphate, hot start enzyme iQTaq DNA polymerase ( 25 units ml$^{-1}$ ), $3 \mathrm{~mm} \mathrm{MgCl} 2$, SYBR Green 1 and $10 \mathrm{~nm}$ fluorescein as a passive reference. Samples of cDNA of $1 \mu \mathrm{l}$ each (assayed in triplicate) was used as a template with $3.2 \mathrm{pmol}$ of primers for p65-NF- $\mathrm{kB}$ (forward, $5^{\prime}$-CATG CGTTTCCGTTACAAGTGCGA- $3^{\prime}$ and reverse $5^{\prime}$-TGGGTGCGTCTTAGTGGT ATCTGT- $3^{\prime}$ ), and $\beta$-actin ( $5^{\prime}$-TCATCACTATCGGCAATGAGCGGT- $3^{\prime}$ and $5^{\prime}$ ACAGCACTGTGTTGGCATAGAGGT- $3^{\prime}$ ) in a final volume of $25 \mu$ l.

The thermal cycling program was $10 \mathrm{~min}$ at $95^{\circ} \mathrm{C}$ followed by 40 cycles of $15 \mathrm{~s}$ at $95^{\circ} \mathrm{C}, 30 \mathrm{~s}$ at $56^{\circ} \mathrm{C}$ and $15 \mathrm{~s}$ at $72^{\circ} \mathrm{C}$. PCR product melting points were determined by incubation at $65^{\circ} \mathrm{C}$ for $1 \mathrm{~min}$, followed by a $1{ }^{\circ} \mathrm{C}$ per min rise over $30 \mathrm{~min}$. The sequence of all primers was confirmed by National Research Institute of Canada, Saskatoon.

\section{Determination of $\mathrm{HO}$ activity and HO-1 concentration}

$\mathrm{HO}$ activity was evaluated as bilirubin production by our established method. $^{27-29}$ In brief, the mesenteric arterioles were homogenized on ice in four volumes of 5:1 K/Na $100 \mathrm{mmoll}^{-1}$ phosphate buffer with $2 \mathrm{mmol} / \mathrm{l}^{-1} \mathrm{MgCl}_{2}$ (HO-activity buffer), centrifuged at 13000 r.p.m. for $15 \mathrm{~min}$. From the supernatant, aliquots of $100 \mu \mathrm{l}$ was to measure $\mathrm{HO}$ enzyme activity in a reaction volume of $500 \mu \mathrm{l}$, containing $0.8 \mathrm{mmoll}^{-1}$ nicotinamide dinucleotide

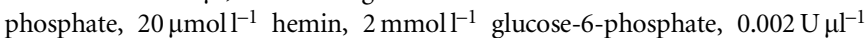
glucose-6-phosphate dehydrogenase and $100 \mu \mathrm{l}$ liver cytosol as source of biliverdin reductase. The reaction was carried out in dark at $37^{\circ} \mathrm{C}$ for $1 \mathrm{~h}$ and stopped by adding $500 \mu \mathrm{l}$ of chloroform. To extract bilirubin, the tubes were vigorously agitated and centrifuged at 13000 r.p.m. for $5 \mathrm{~min}$. The chloroform layer was collected and read on a spectrophotometer at $464 \mathrm{~nm}$ minus the background at $530 \mathrm{~nm}$. The amount of bilirubin in each sample was determined spectrophotometrically (extinction coefficient for bilirubin $40 \mathrm{~mm}^{-1} \mathrm{~cm}^{-1}$ ), and expressed as nmol per mg protein per hour. As a positive control, spleen tissue was used. HO-1 concentration was determined by enzyme-linked immunosorbent assay (EKS-810A, Stressgen-Assay Design, Ann Arbor, MI, USA) as previously reported. ${ }^{27}$

\section{Determination of 8-isoprostane}

8 -isoprostane is a non-invasive index of oxidative stress. This was determined using enzyme immunoassay (EIA; Cayman Chemical Company, Ann Arbor, MI, USA) as we previously described. ${ }^{27,28}$ In brief, the mesenteric arterioles were homogenized in phosphate buffer containing $0.005 \%$ butylated hydroxy toluene in a ratio of $10 \mu \mathrm{l}$ buffer per mg tissue. Subsequently, an equal volume of $15 \% \mathrm{KOH}$ was added to the homogenate. The samples were incubated at $40{ }^{\circ} \mathrm{C}$ for $1 \mathrm{~h}$, followed by centrifugation, and the supernatant was neutralized with $\mathrm{KH}_{2} \mathrm{PO}$ and the diluted 1:15 with ultrapure water, applied to a reversephase C-18 column at $\mathrm{pH} 3$, and eluted with 1:1 ( vol vol $^{-1}$ ) ethyl acetate/ heptane. Thereafter, the eluent was further purified on a silica column and eluted with 1:1 ( $\left.\mathrm{vol} \mathrm{vol}^{-1}\right)$ ethylacetate/methanol, and aliquoted into plates precoated with monoclonal antibody. After 8-isoprostane tracer and isoprostane antiserum were added to each well and incubated and after washing, Ellman's reagent containing the substrate of acetylcholinesterase was added. The absorbance were read at $412 \mathrm{~nm}$ in a plate reader (SpectraMax 340PC; Molecular Device, Sunnyvale, CA, USA), and the values of 8-isoprostane were calculated from a standard curve. The levels of mesenteric arteriole 8 -isoprostane were expressed as pg per $\mathrm{mg}$ of protein.

\section{Determination of ET-1}

ET-1 was quantified using EIA (Cayman Chemical) according to the manufacturer's instructions. This immunometric assay is based on a double-antibody 'sandwich' technique that detects ET-1 within the range of $0-250 \mathrm{pg} \mathrm{ml}^{-1}$. In brief, supernatants from homogenized tissues and previously obtained 
plasma were purified by cold spike extraction, concentrated and the absorbance read at $405 \mathrm{~nm}$ in a plate reader (SpectraMax 340PC; Molecular Device).

\section{PLC activity assay}

The PLC was determined using a PLC assay kit (Cayman Chemical), as we previously reported. ${ }^{28}$ In this assay, the substrate arachidonyl thio-phosphatidylcholine generates DTNB (5,5'-dithiobis-2-dinitrobenzoic acid) that is detected and quantified. ${ }^{33}$ In brief, mesenteric arterioles were homogenized, centrifuged, and the supernatant was treated with thioetaramide-phosphatidylcholine and bromoenol lactone to block secretory phospholipase A and calcium-independent phospholipase A and incubated with arachidonyl thiophosphatidylcholine. Subsequently, DTNB/EGTAethylene glycol tetraacetic acid) was added to each sample and read at $414 \mathrm{~nm}$ with a microplate reader (SpectraMax 340PC; Molecular Device). PLC activity was calculated from absorbance. PLC-positive controls were used to ascertain accuracy.

\section{Determination of $\mathrm{IP}_{3}$ concentration}

$\mathrm{IP}_{3}$ was quantified using a sensitive D-myo-IP 3 Biotrak Assay System, $\left({ }^{3} \mathrm{H}\right.$-TRK1000 kit; Amersham International, Amersham, Buckinghamshire, UK) as we previously reported. ${ }^{28}$ In brief, the mesenteric arterioles were homogenized in 0.2 volume ice-cold $20 \%$ percloric acid, centrifuged at $2000 \mathrm{~g}$ and the supernatant decanted and neutralized to $\mathrm{pH} 7.5$ with ice-cold $10 \mathrm{M} \mathrm{NaOH}$, and was again centrifuged and the supernatant recovered and $\mathrm{IP}_{3}$ detected following instructions from the manufacturer and expressed as nmol $\min ^{-1} \mathrm{ml}^{-1}$.

\section{Measurement of cGMP content}

The concentration of cyclic guanosine monophosphate (cGMP) was evaluated using EIA (Cayman Chemical) as we previously reported. ${ }^{28,30}$ In brief, the mesenteric arterioles were homogenized in $6 \%$ trichloroacetic acid at $4{ }^{\circ} \mathrm{C}$ in the presence of $3^{\prime}$-isobutyl-1-methylxanthine to inhibit phosphodiesterase activity and centrifuged at $2000 \mathrm{~g}$ for $15 \mathrm{~min}$. The supernatant was recovered and washed three times with water-saturated diethyl ether. The upper ether layer was aspired and discarded whereas the aqueous layer containing cGMP was recovered and lyophilized. The dry extract was dissolved in assay buffer and the cGMP content was measured using the manufacturer's protocol and expressed as pmol of cGMP per mg of protein.

\section{Determination of intracellular calcium}

The concentration of intracellular $\mathrm{Ca}^{2+}$ was measured by our established method. $^{28,34}$ In brief, vascular smooth muscle cells from the mesenteric arterioles were isolated and incubated with $4 \mu \mathrm{M}$ Fluo 3-acetoxymethyl ester and $0.08 \%$ pluronic $\mathrm{F}-127$ at $37^{\circ} \mathrm{C}$ for $30 \mathrm{~min}$ in Dulbecco's modified Eagle's medium containing $10 \%$ fetal bovine serum. The cells were centrifuged, suspended in Dulbecco's modified Eagle's medium, and later in Hank's balanced salt solution containing $1 \mathrm{mg} \mathrm{m}^{-1}$ bovine serum albumin. Approximately $5 \times 10^{4}$ cells were transferred in each well and incubated at $37^{\circ} \mathrm{C}$ for $5 \mathrm{~min}$. The fluorescence of Fluo-3 was measured for $10 \mathrm{~min}$ with filters for excitation at $485 \mathrm{~nm}$ and for emission at $527 \mathrm{~nm}$, using a Microplate Fluorometer and Ascent software (Thermo Labsystems, Beverly, MA, USA).

\section{Determination of superoxide dismutase activity}

Superoxide dismutase activity was determined using a commercial EIA assay kit (Cayman Chemical) as we previously reported. ${ }^{27,28}$ In brief, the mesenteric arterioles were homogenized in a solution containing 20 mM HEPES at $\mathrm{pH} 7.2$, $1 \mathrm{~mm}$ EGTA, $210 \mathrm{~mm}$ mannitol and $70 \mathrm{~mm}$ sucrose per g tissue, and centrifuged at $1500 \mathrm{~g}$ for $5 \mathrm{~min}$ at $4{ }^{\circ} \mathrm{C}$. The supernatant was recovered, and superoxide dismutase activity was calculated from a standard curve generated from reading the absorbance at $450 \mathrm{~nm}$ with a microplate reader (SpectraMax 340PC; Molecular Device).

\section{Total anti-oxidant capacity assay}

The total anti-oxidant capacity was evaluated using EIA Kit (Cayman Chemical) as we previously reported. ${ }^{27,28}$ In tissues, the antioxidant system comprises different enzymes, including superoxide dismutase, catalase and glutathione peroxidise, as well as substances such as ferritin, ascorbic acid, $\alpha$-tocopherol, $\beta$-carotene, reduced glutathione, uric acid, biliverdin and bilirubin. ${ }^{21-26,35}$ The sum of endogenous and food-derived antioxidants represents the total antioxidant activity of the system. The additive effect of all the different antioxidants provides greater protection against oxidative stress than any single compound alone.

The Cayman Chemical Antioxidant Assay Kit measures the total antioxidant capacity. This assay relies on the ability of the anti-oxidants in samples to inhibit the oxidation of ABTS $\left(2,2^{\prime}\right.$-azino-di-(3-ethylbenzthiazoline sulphonate) to ABTS plus metmyoglobin. In brief, the mesenteric arterioles were homogenized in the presence of protease inhibitors and treated with Trolox (6-hydroxy-2, 5, 7, 8-tetramethylchroman-2-carboxylic acid), metmyoglobin and chromogen. The reaction is initiated by adding $\mathrm{H}_{2} \mathrm{O}_{2}$ and after $5 \mathrm{~min}$ of incubation, the absorbance is read at $750 \mathrm{~nm}$ using Synergy Microplate Reader with Gen5 Data Analysis Software (BioTek, Winooski, VT, USA), and expressed as Trolox equivalent antioxidant capacity per mg protein.

\section{In vitro treatment of mesenteric arterioles with carbon monoxide-} releasing molecule

In another experimental setting, vascular smooth muscle cells from the mesenteric arterioles were isolated from several groups, including (A) surgery-free SD control, (B) UnX-sham, (C) UnX-DOCA and (D) UnX DOCAsalt and pre-treated with the carbon monoxide (CO)-releasing molecule (CORM-3) to ascertain the role of $\mathrm{CO}$ in the heme-arginate-induced effects on NF- $\mathrm{KB}$, cGMP, ET-1, PLC, $\mathrm{IP}_{3}$ and resting intracellular calcium.

Tricarbonyldichlororuthenium(II) dimer $\left(\left[\mathrm{Ru}(\mathrm{CO})_{3} \mathrm{Cl}_{2}\right]_{2}\right)$ and dimethylsulfoxide were obtained from Sigma-Aldrich. After dissolving $\left[\mathrm{Ru}(\mathrm{CO})_{3} \mathrm{Cl}_{2}\right]_{2}$ in $100 \%$ dimethylsulfoxide, tricarbonylchloro(glycinato)ruthenium(II) ( $\left[\mathrm{Ru}(\mathrm{CO})_{3} \mathrm{Cl}\right.$ (glycinate) $]$ or CORM-3 was prepared by adding glycine as described previously. ${ }^{36}$ Stock solutions of CORM-3 $\left(0.11 \mathrm{moll}^{-1}\right)$ were prepared by dissolving the compound in distilled water. As a negative control, inactive CORM-3 (iCORM-3) was prepared by dissolving CORM-3 in phosphate-buffered saline and allowing the liberation of $\mathrm{CO}$ for $18 \mathrm{~h}$ at $37^{\circ} \mathrm{C}$ in a fume hood. Subsequently, the iCORM-3 compound was bubbled with nitrogen to remove any residual $\mathrm{CO}$.

After preparing CORM-3, isolated vascular smooth muscle cells from the mesenteric arterioles of the different experimental groups were pre-incubated with $200 \mu \mathrm{M}$ of CORM-3 or iCORM-3 for $1 \mathrm{~h}$ at $37^{\circ} \mathrm{C}$ before proceeding with the different assays for cGMP, ET-1, NF- $\mathrm{KB}, \mathrm{PLC}, \mathrm{IP}_{3}$ and resting intracellular calcium. We used $200 \mu \mathrm{M}$ of CORM-3 because this dose was reportedly effective in in vitro studies. ${ }^{37}$

\section{Statistical analysis}

All data were expressed as means \pm s.e.m. and analyzed using Student's $t$-test and analyses of variance in conjunction with Bonferroni's test for multiple comparisons. Group differences at the level of $P<0.05$ are statistically significant.

\section{RESULTS}

Heme-arginate reduced blood pressure in DOCA-salt hypertensive rats

UnX-DOCA-salt rats were severely hypertensive of $197.8 \pm 4.6 \mathrm{~mm} \mathrm{Hg}$ whereas the control animals, including UnX-sham, UnX-salt, UnXDOCA and surgery-free SD rats, were normotensive with BP readings of $120.5 \pm 3.6,120.8 \pm 4.1,121.7 \pm 3.6$ and $119.8 \pm 4.3 \mathrm{~mm} \mathrm{Hg}$, respectively. As expected, the 4-week therapy of heme-arginate lowered blood pressure in DOCA-salt hypertensive rats (197.8 \pm 4.6 vs. $132.5 \pm 3.5 \mathrm{~mm} \mathrm{Hg} ; \quad P<0.01$; Table 1), whereas blood pressure remained elevated in heme-arginate+CrMP+UnX-DOCA-salt group $(209.7 \pm 5.6 \mathrm{~mm} \mathrm{Hg})$ and was exacerbated in the CrMP+UnX-DOCAsalt group $(224.8 \pm 8.5 \mathrm{~mm} \mathrm{Hg})$, suggesting a role of basal $\mathrm{HO}$ in the regulation of blood pressure. After the first week of treatment, BP dropped from $197.8 \pm 4.6$ to $179.5 .4 \pm 4.1 \mathrm{~mm} \mathrm{Hg}$, and decreased 
gradually to $161.5 \pm 3.5 \mathrm{~mm} \mathrm{Hg}$ by the end of the second week. Thereafter, blood pressure progressively decreased to $142.6 \pm 3.7$ and $132.5 \pm 3.5 \mathrm{~mm} \mathrm{Hg}$ after the third and fourth week, respectively. Heme-arginate also decreased blood pressure slightly in normal SD rats $(119.8 \pm 4.3$ vs. $110.4 \pm 2.7 \mathrm{~mm} \mathrm{Hg} ; P<0.05)$. In contrast, the vehicle had no effect on blood pressure in UnX-DOCA-salt hypertensive rats $(197.8 \pm 4.6$ vs. $201.5 \pm 5.2 \mathrm{~mm} \mathrm{Hg})$.

During the 4 -week treatment period, a slight body weight loss $(7.6 \%)$ was observed in the UnX-DOCA-salt+heme-arginate+CrMP group (Table 1). This may be due to the stress of polytherapy of hemearginate and CrMP, as injecting the drugs individually did not cause body weight loss.

Heme-arginate upregulates the HO system and the cGMP signaling To evaluate the cytoprotective effects of heme-arginate, we measured HO- $1, \mathrm{HO}$ activity and cGMP. The basal HO- 1 and HO-activity in the controls (surgery-free or normal SD rats, UnX-sham, UnX-salt and UnX-DOCA) were comparable, but lower than the levels in UnXDOCA-salt hypertensive rats. Heme-arginate increased HO- 1 and $\mathrm{HO}$ activity in UnX-DOCA-salt hypertensive rats by 2.5- and 3.2-fold, respectively (Figures $1 \mathrm{a}$ and $\mathrm{b}$ ), whereas co-treatment with the $\mathrm{HO}$ inhibitor, CrMP, nullified the effect of heme-arginate. Similarly, treatment with CrMP alone reduced the basal HO-1 and $\mathrm{HO}$ activity (Figures $1 \mathrm{a}$ and $\mathrm{b}$ ). The enhanced $\mathrm{HO}$ activity would increase endogenous carbon monoxide production that would in turn stimulate the production of cGMP. ${ }^{30}$ Accordingly, we detected a 2.7-fold increase of cGMP in heme-arginate-treated animals (Figure 1c). On the other hand, the effect of heme-arginate on cGMP was abolished in the CrMP+heme-arginate group, whereas a further decrease in cGMP was observed in the animals treated with CrMP alone. Although the basal HO activity in DOCA-salt hypertensive rats was higher than the controls, it did not evoke an increase in cGMP content (Figure 1c). The reason for this is not fully understood and needs to be clarified by further studies. However, a possible explanation could be that the magnitude of basal HO in DOCA-salt hypertensive rats might have fallen below the threshold necessary to trigger an increase in cGMP content. A similar observation has been previously reported. ${ }^{27,30}$

Heme-arginate also enhanced HO-1, HO activity and cGMP in SD rats, although a greater increment was observed in heme-arginatetreated DOCA-salt animals (Figure 1). The higher magnitude of $\mathrm{HO}$ signaling may be responsible for the more intense effect on blood pressure in DOCA-salt rats when compared with SD rats (Table 1). Alternatively, the less-preponderant increase of $\mathrm{HO}$ activity in SD rats may suggest greater stability of the HO system in normal conditions.

Heme-arginate enhanced the anti-oxidant status in DOCA-salt rats As anti-oxidants such as bilirubin and ferritin are generated by the $\mathrm{HO}$ system, ${ }^{21,22,26}$ and superoxide dismutase is modulated HO- $1,{ }^{38}$ we analyzed the effect of upregulating the HO system on these antioxidants. In UnX-DOCA-salt rats, the basal levels of bilirubin, ferritin and superoxide dismutase were significantly reduced (Figures $2 \mathrm{a}-\mathrm{c}$ ), and were increased by heme-arginate by 1.92, 2.77 and 1.91-fold, respectively, whereas CrMP abolished the effect of heme-arginate on these anti-oxidants. Interestingly, the heme-arginate-induced increment of bilirubin, ferritin and superoxide dismutase was accompanied by significant elevation of the total anti-oxidant capacity (Figure $2 \mathrm{~d}$ ). In UnX-DOCA hypertensive rats, the basal level of the total antioxidant capacity was threefold lower than the controls, but was enhanced by heme-arginate to control levels. In the heme-arginate + CrMP group, the total anti-oxidant capacity was reversed to the 

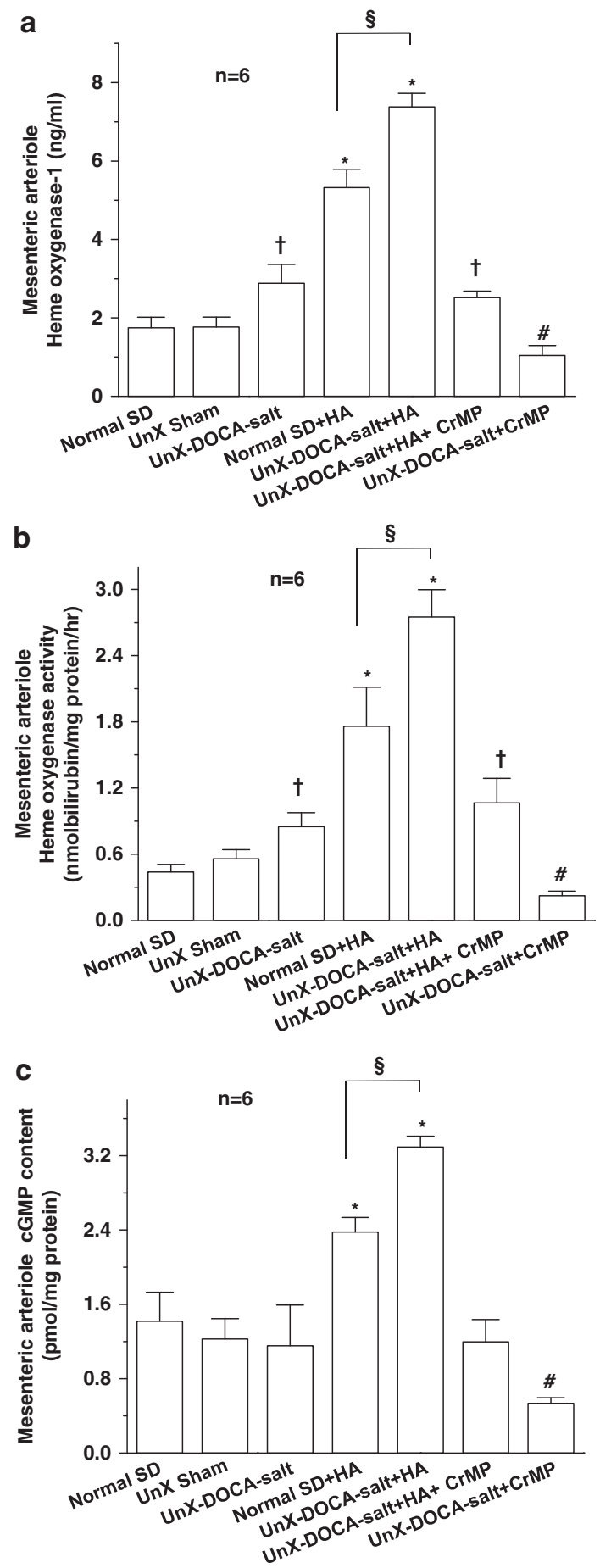

Figure 1 Effects of the $\mathrm{HO}$ inducer, heme-arginate, and the $\mathrm{HO}$ inhibitor, CrMP, on HO-1, HO activity and CGMP in the mesenteric arterioles of UnXDOCA-salt hypertensive rats. The basal (a) HO-1 concentration, and (b) $\mathrm{HO}$ activity in UnX-DOCA-salt hypertensive rats was higher than in the controls. Heme-arginate greatly increased $\mathrm{HO}$ activity in DOCA-salt hypertensive rats, but the HO blocker, CrMP, nullified the effect of heme-arginate. (c) Treatment with heme-arginate markedly increased cGMP content of DOCA-salt hypertensive rats, whereas CrMP abolished the effect. The bars represent means \pm s.e.; $n=6$ rats per group $\left({ }^{*} P<0.01\right.$ vs. all groups; ${ }^{\dagger} P<0.05$ vs. all groups; ${ }^{\sharp} P<0.05$ vs. all groups; ${ }^{\S} P<0.05$ ). levels of UnX-DOCA-salt hypertensive rats, and further reduced in CrMP-treated animals to lower levels, suggesting that inhibiting the HO system may lead to the suppression of anti-oxidant defense mechanisms.

Heme-arginate also enhanced the levels of bilirubin, ferritin and superoxide dismutase, which, interestingly, was accompanied by the parallel potentiation of the total anti-oxidant capacity in SD rats (Figures $2 \mathrm{a}-\mathrm{d}$ ). However, the magnitude of the increment of bilirubin, ferritin and superoxide dismutase was less intense in SD rats when compared with UnX-DOCA-salt hypertensive rats, suggesting greater selectivity of the HO system in a diseased condition such as mineralocorticoid-induced hypertension.

\section{Heme-arginate abates 8-isoprostane, ET-1 and NF-кB in the} mesenteric arteriole of DOCA-salt hypertension

As the mechanisms by which ET-1 triggers oxidative insults include stimulating the formation of 8-isoprostane, ${ }^{3}$ and activating the nuclear translocation and transcriptional activity of NF- $\kappa \mathrm{B},{ }^{39,40}$ we analyzed whether the heme-arginate-induced potentiation of the total-antioxidant status in UnX-DOCA-salt hypertensive rats would affect important mediators of oxidative injury such as 8-isoprostane, ET-1 and NF- $\kappa$ B. Interestingly, the potentiation of the antioxidant status in UnX-DOCA-salt hypertensive rats was accompanied by a marked and parallel reduction of 8 -isoprostane, which is a marker of oxidative stress (Figure 3a). In UnX-DOCA-salt hypertensive rats, the elevated levels of 8 -isoprostane were reduced by 3.6-fold, whereas CrMP abolished the effects of heme-arginate, exacerbating 8-isoprostane, and thus oxidative stress. Similarly, heme-arginate suppressed the elevated ET-1 levels in UnX-DOCA-salt hypertensive rats by 2.9-fold (Figure $3 \mathrm{~b}$ ), whereas the HO inhibitor, CrMP, abolished the effects of heme-arginate, restoring the high ET-1 levels in UnX-DOCA-saltheme-arginate+CrMP, and further exasperated ET-1 levels in UnXDOCA-salt+CrMP, suggesting an important role of the basal HO system in the regulation of ET-1 in mineralocorticoid-induced hypertension. Analogically, the increased NF- $\mathrm{kB}$ mRNA expression in UnXDOCA-salt hypertensive rats was reduced by 1.93 -fold (Figure $3 \mathrm{~b}$ ). In contrast, CrMP abolished the heme-arginate-mediated reduction of NF- $\kappa B$, restoring elevated levels of NF- $\kappa B$ in UnX-DOCA-saltheme-arginate+CrMP and UnX-DOCA-salt+CrMP, which, interestingly, were comparable to the levels observed in UnX-DOCA-salt hypertensive rats (Figure $3 \mathrm{~b}$ ).

Heme-arginate also reduced the levels of 8-isoprostane, ET-1 and $\mathrm{NF}-\kappa \mathrm{B}$ in normotensive SD rats. Our results indicate that hemearginate further depleted the reduced levels of 8-isoprostane, ET-1 and $\mathrm{NF}-\kappa \mathrm{B}$ in $\mathrm{SD}$ rats (Figures $3 \mathrm{a}-\mathrm{c}$ ). However, the reduction was less intense in SD rats when compared with UnX-DOCA-salt hypertensive rats, suggesting greater selectivity of the $\mathrm{HO}$ system against 8-isoprostane, ET-1 and NF- $\mathrm{KB}$ in mineralocorticoid-induced hypertension.

Heme-arginate abates the $\mathrm{PLC}-\mathrm{IP}_{3}$ signaling and reduces resting intracellular calcium of the mesenteric arterioles

One of the major signal transduction mechanisms for the mobilization of intracellular calcium and the control of vascular smooth muscle contractility is the PLC-IP $3.1{ }^{1,41}$ The basal PLC activity in UnXDOCA-salt hypertensive rats was significantly higher than in the controls, but was greatly reduced by heme-arginate (Figure 4a), although control values were not reinstated. Importantly, a 2.4-fold reduction in PLC was observed in heme-arginate-treated animals. Similarly, $\mathrm{IP}_{3}$ activity was abrogated by 3.3 -fold by heme-arginate (Figure $4 \mathrm{~b}$ ). Contrarily, in the heme-arginate+CrMP group, PLC and 

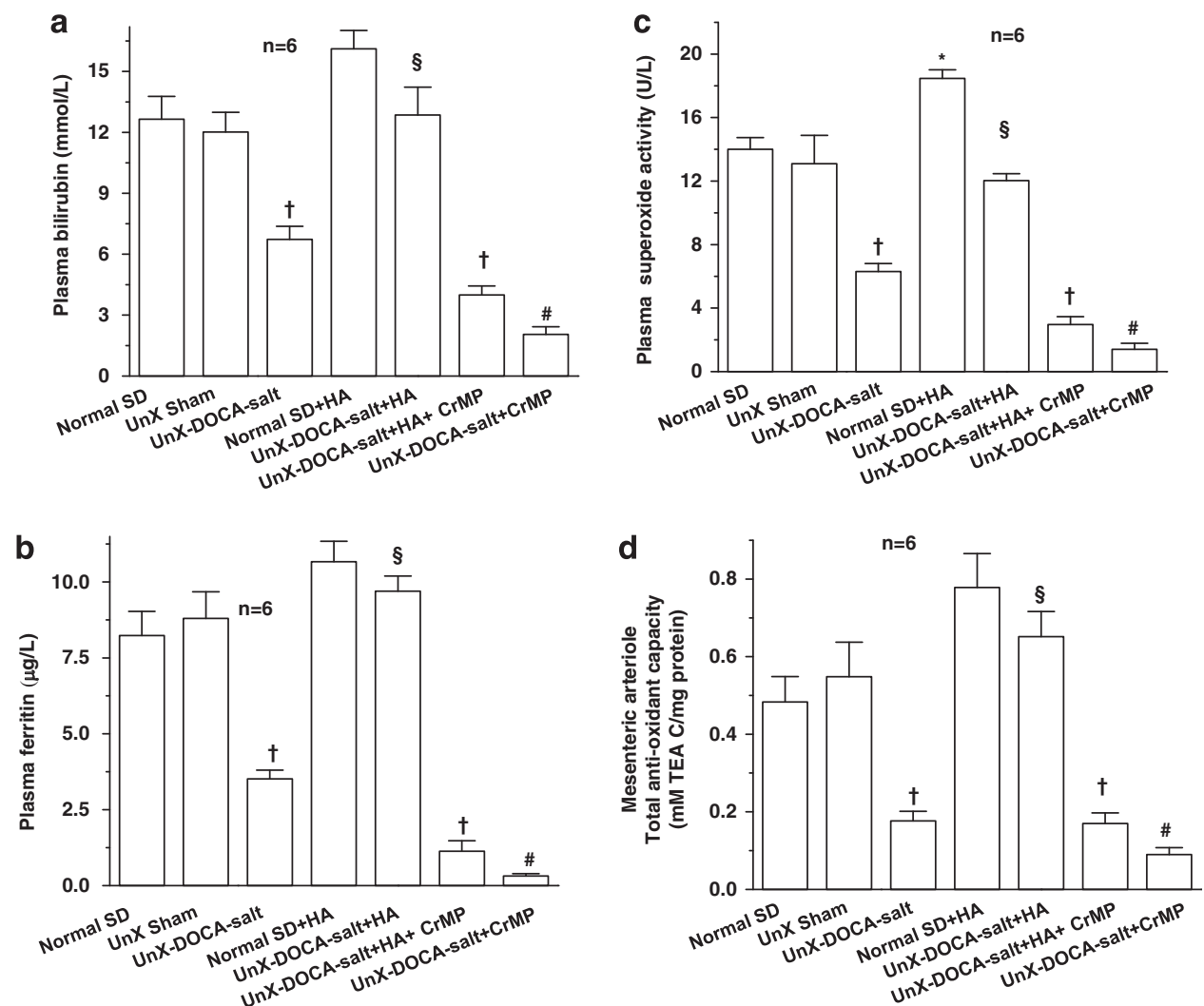

Figure 2 Effects of heme-arginate and CrMP on anti-oxidants in UnX-DOCA-salt hypertensive rats. Heme-arginate enhanced the levels of plasma (a) bilirubin, (b) ferritin and (c) superoxide dismutase activity, and (d) mesenteric arteriole total-anti-oxidant capacity, whereas CrMP nullified the effects of heme-arginate. The bars represent means \pm s.e.; $n=6$ rats per group $\left({ }^{*} P<0.05\right.$ vs. all groups; ${ }^{\dagger} P<0.05$ vs. all groups; ${ }^{\#} P<0.05$ vs. all groups; ${ }^{\circledR} P<0.01$ vs. UnX-DOCAsalt).

$\mathrm{IP}_{3}$ were reversed to the high levels observed in UnX-DOCA-salt hypertensive rats, and the levels were further increased in CrMPtreated animals, suggesting a role of the $\mathrm{HO}$ system in the regulation of PLC-IP 3 signaling. Interestingly, the suppression of the PLC-IP3 signaling was accompanied by a significant reduction in resting intracellular calcium concentration (Figure 4c), although control levels were not attained. Importantly, heme-arginate reduced the resting calcium concentration by 2.2 -fold, whereas the HO inhibitor, CrMP, abolished the heme-arginate-induced reduction and exacerbated the levels of resting calcium.

Heme-arginate also reduced PLC and $\mathrm{IP}_{3}$ activities, and resting intracellular calcium concentration in SD rats, although the effect was less intense when compared with UnX-DOCA-salt hypertensive rats (Figure 4c), suggesting greater selectivity of heme-arginate against the PLC-IP ${ }_{3}$-resting intracellular calcium signaling in mineralocorticoidinduced hypertension. It is worth mentioning that the suppression of the PLC-IP $\mathrm{I}_{3}$ secondary messenger signal transduction pathway in UnX-DOCA-salt hypertensive rats by heme-arginate is an important observation.

\section{CORM-3 abates cGMP, ET-1, NF- $\kappa \mathrm{B}, \mathrm{PLC}, \mathrm{IP}_{3}$ and resting} intracellular calcium of the mesenteric arterioles

To ascertain the involvement of $\mathrm{CO}$ in the heme-arginate-induced effects, isolated mesenteric arterioles from several groups including surgery-free or normal SD rats, UnX-sham, UnX-DOCA and UnX DOCA-salt groups were pre-treated with the active CORM-3 and its inactive form, iCORM-3, before assaying cGMP, ET-1, NF- $\kappa \mathrm{B}$, PLC, $\mathrm{IP}_{3}$ and resting intracellular calcium. Our results indicate that the basal levels of cGMP in normotensive controls (surgery-free SD rats, UnXsham and UnX-DOCA) were comparable to UnX-DOCA-salt hypertensive rats (Figure 5a). Interestingly, CORM-3 significantly enhanced cGMP content in UnX-DOCA-salt hypertensive rats by 2.6-fold, whereas iCORM-3 had no effect as it was devoid of CO-releasing capability. Similarly, CORM-3 ablated the levels of ET-1, NF- $\kappa$ B, PLC, $\mathrm{IP}_{3}$ by 2.4-, 1.8-, 2.5- and 3.3-fold, respectively, with a corresponding reduction in resting intracellular calcium of the mesenteric arterioles (Figures $5 \mathrm{~b}-\mathrm{f}$ ), in a manner that is consistent with the observations in heme-arginate-treated animals. In contrast, iCORM-3 had no effect on any of the measured parameters. It is noteworthy that although CORM-3 abrogated the levels of cGMP, ET-1, NF- $\kappa B$, PLC, $\mathrm{IP}_{3}$ and resting intracellular calcium in UnX-DOCA-salt hypertensive rats, it failed to reinstate similar levels as observed in the normotensive controls.

\section{DISCUSSION}

In this present study, the administration of heme-arginate to UnXDOCA-salt hypertensive rats concomitantly suppressed 8-isoprostane, ET-1, NF- $\kappa \mathrm{B}$ and the PLC-IP ${ }_{3}$ signal transduction pathway. This was associated with a corresponding reduction in oxidative/inflammatory insults alongside the decline in resting intracellular calcium in smooth muscle cells of the mesenteric arterioles and the restoration of physiological blood pressure. Similarly, treatment of mesenteric arterioles in vitro with CORM-3 resulted in potentiating cGMP signaling 

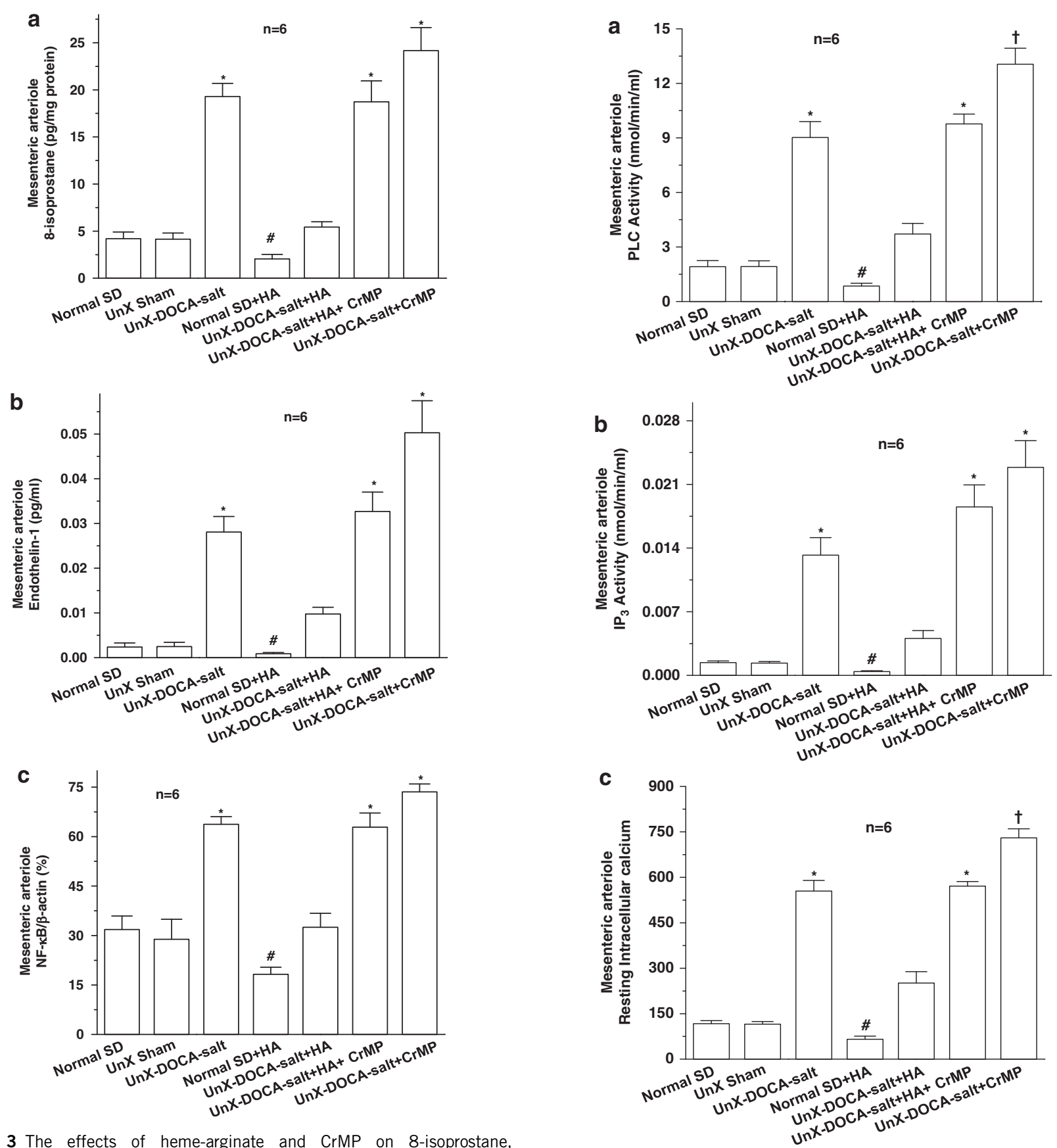

Figure 3 The effects of heme-arginate and CrMP on 8-isoprostane, endothelin-1 and NF-KB in the mesenteric arterioles of UnX-DOCA-salt hypertensive rats. Heme-arginate markedly reduced (a) 8-isoprostane and (b) endothelin-1, but the HO blocker, CrMP, annulled the effects of hemearginate. (c) Quantitative real-time reverse transcriptase PCR (RT-PCR) indicated that heme-arginate suppressed the elevated basal mRNA expression of NF-KB, but the effect of heme-arginate was nullified by CrMP. The bars represent means \pm s.e.; $n=6$ rats per group $\left({ }^{*} P<0.01\right.$ vs. all groups; ${ }^{\#}<<0.05$ vs. all groups).

but abating ET-1, NF- $\mathrm{\kappa B}, \mathrm{PLC}, \mathrm{IP}_{3}$ and resting intracellular calcium levels. In UnX-DOCA-salt hypertensive rats, elevated ET-1 and high levels of the synthetic mineralcorticoid (DOCA) led to excessive oxidative stress. ${ }^{1-4}$ Accordingly, the high levels of 8 -isoprostane detected in UnX-DOCA-salt hypertensive rats is a testimony of the highly oxidative milieu that characterizes this model.

Besides inducing oxidative stress, the combined effects of PLC- $\mathrm{IP}_{3}$ signaling, ET-1 and 8-isoprostane $\mathrm{e}^{2,3}$ constitute a potent vasoconstrictor force that would trigger blood pressure elevation and promote tissue injury by activating the oxidative/inflammatory transcription factor, NF- $\kappa \mathrm{B} .{ }^{14,15,42}$ Interestingly, the application of heme-arginate upregulated the $\mathrm{HO}$ system and enhanced several anti-oxidants,

Figure 4 The effects of heme-arginate and CrMP on PLC, IP 3 and resting intracellular calcium levels in the mesenteric arterioles of UnX-DOCA-salt hypertensive rats. Heme-arginate significantly reduced (a) PLC activity and (b) $\mathrm{IP}_{3}$ activity and (c) resting intracellular calcium levels, but the $\mathrm{HO}$ blocker, CrMP, cancelled the heme-arginate effects. The bars represent means \pm s.e.; $n=6$ rats per group ( ${ }^{*} P<0.01$ vs. all groups; ${ }^{\dagger} P<0.05$ vs. all groups; ${ }^{\#} P<0.05$ vs. all groups). 
a

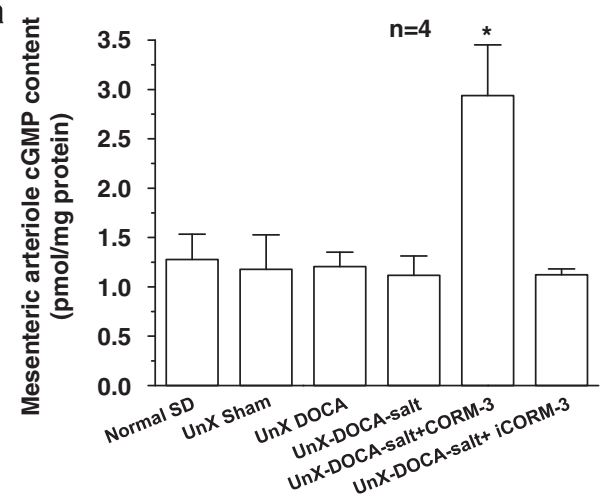

b

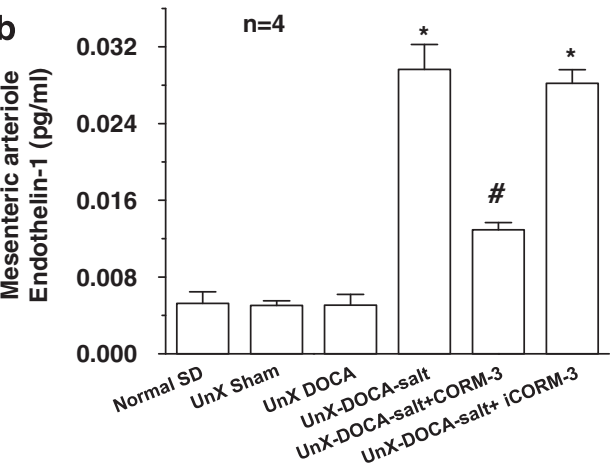

C

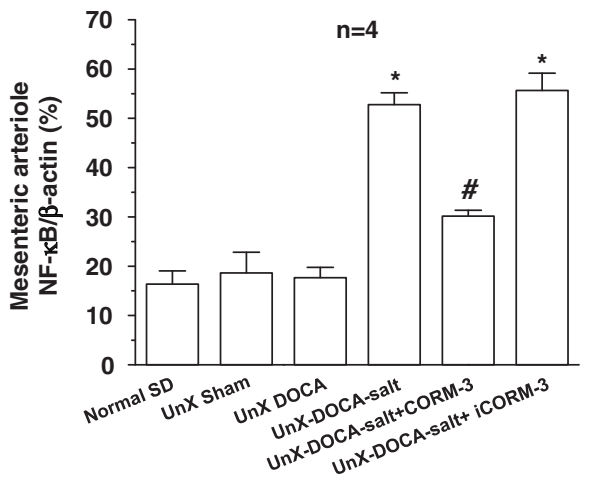

d 6 [ $n=4$
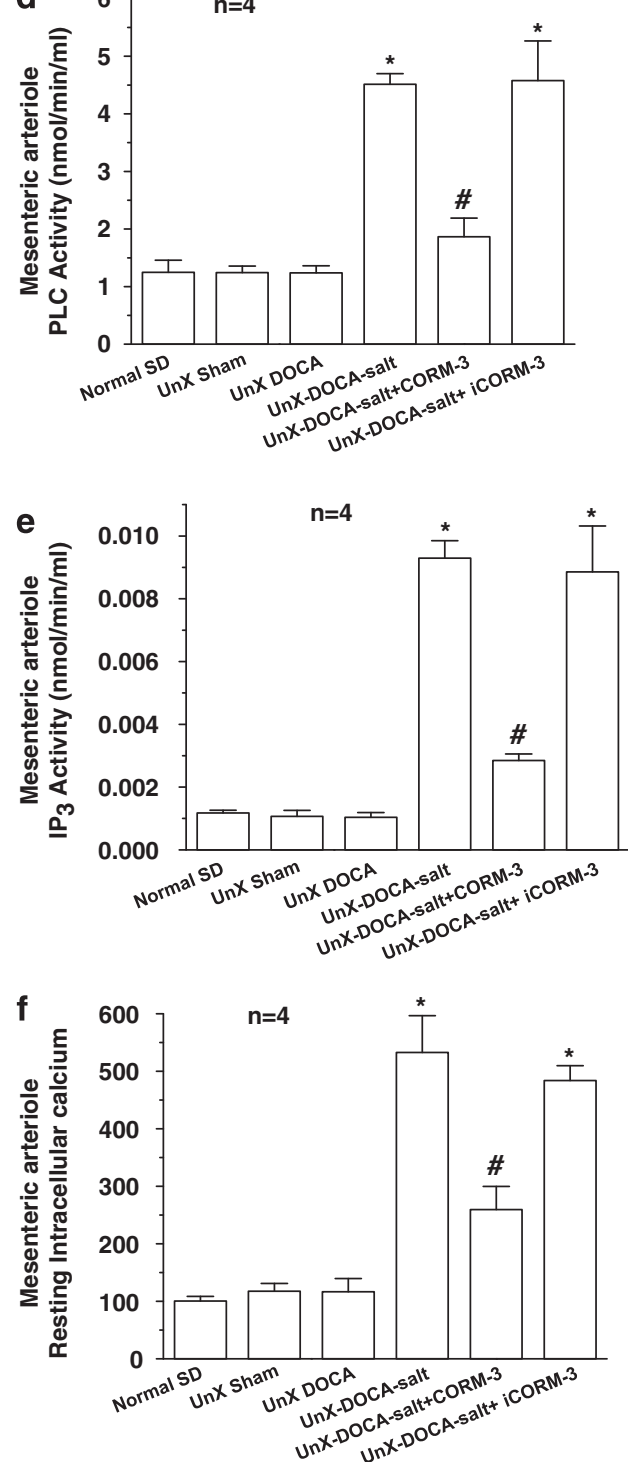

Figure 5 The effects of the CO-releasing molecule, CORM-3, on cGMP, ET-1, NF- $\mathrm{kB}, \mathrm{PLC}, \mathrm{IP}_{3}$ and resting intracellular calcium in the mesenteric arterioles of UnX-DOCA-salt hypertensive rats. CORM-3 markedly attenuated (a) CGMP, (b) ET-1, (c) NF- $\mathrm{KB}$, (d) PLC activity, (e) IP 3 activity and (f) resting intracellular calcium levels, whereas iCORM-3 had no effect. The bars represent means \pm s.e.; $n=4$ rats per group $\left({ }^{*} P<0.01\right.$ vs. all groups; ${ }^{\#} P<0.05$ vs. all groups).

including bilirubin, ferritin and superoxide dismutase, with the potentiation of the total anti-oxidant capacity. Correspondingly, marker/ mediators of oxidative injury, such as 8-isoprostane and $\mathrm{NF}-\kappa \mathrm{B}$, were significantly depleted, whereas the HO inhibitor, CrMP, abolished the protective effects of heme-arginate and exacerbated blood pressure and oxidative/inflammatory insults. These observations are consistent with our recent studies in which upregulating the $\mathrm{HO}$ system with hemin or heme-arginate normalized blood pressure and abated oxidative/inflammatory mediators such as 8-isoprostane, NF$\kappa \mathrm{B}$ and activating proteins 1 and 2, with the attenuation of cardiac and renal histopathological lesions in different models, including spontaneously hypertensive rats and UnX-DOCA-salt hypertension. . $^{27,28,31,43}$ Similarly, many studies have underscored the cytoprotective effect of the HO system against hemorrhagic shock, ${ }^{44}$ corneal inflammation, ${ }^{19}$ insulin resistance, ${ }^{19}$ arthrosclerosis and nephrotoxicity. ${ }^{19}$

Although the cytoprotective effects of HO inducers have been well acknowledged, ${ }^{19,45}$ little is known about the effects of heme-arginate on the PLC- $\mathrm{IP}_{3}$ pro-hypertensive axis in UnX-DOCA-salt hypertensive rats. Our study unveils the modulatory role of HO on the PLC$\mathrm{IP}_{3}$ signal transduction pathway in this model. The PLC- $\mathrm{IP}_{3}$ is an important secondary messenger system that mobilizes intracellular calcium and thus controls vascular tone and blood pressure. ${ }^{11,41}$ Consistently, the suppression of the PLC- $\mathrm{IP}_{3}$ pathway observed in our study is in agreement with reports showing the attenuation of PLC activity in isolated guinea pig coronary smooth muscle cells in vitro. ${ }^{46,47}$ The simultaneous reduction of PLC activity, ET-1 and NF$\kappa \mathrm{B}$ by an upregulated $\mathrm{HO}$ system will attenuate tissue inflammation. ${ }^{44,45,48}$ This notion is consistent with previous studies in which an upregulated HO system suppressed immune/inflammatory responses by abolishing macrophage infiltration and reducing mast-cell and basophils histamine release. ${ }^{45}$ Given that NF- $\kappa \mathrm{B}$ is activated by PLC, ${ }^{15,16}$ and PLC mobilizes intracellular calcium to elevate blood pressure ${ }^{11}$ and stimulate inflammation, ${ }^{48}$ upregulating the HO system would be beneficial in these conditions. 
An important observation from our study is that in vitro treatment of mesenteric arterioles with CORM-3 mimicked the effects of heme-arginate. Although both heme-arginate and CORM-3 abrogated the levels of cGMP, ET- $1, \mathrm{NF}-\kappa \mathrm{B}, \mathrm{PLC}, \mathrm{IP}_{3}$ and resting intracellular calcium in UnX-DOCA-salt hypertensive rats, heme-arginate was more effective than CORM-3. This may be due to the generation of many protective products by the $\mathrm{HO}$ system. Indeed, the induction of $\mathrm{HO}$ by heme-arginate will enhance the yield of other cytoprotective products, such as bilirubin and ferritin, which might contribute in the overall protection conferred by heme-arginate. In addition, the contribution of nitric oxide cannot be ruled out because L-arginine, an ingredient of heme-arginate, also yields nitric oxide, a vasoactive gas that has many protective features similar to CO.

Heme-arginate also upregulated the $\mathrm{HO}$ system and its related signaling pathways in normotensive SD rats, although the magnitude was smaller when compared with UnX-DOCA-salt hypertensive rats. The reasons for this selective effect of $\mathrm{HO}$ are not fully understood. However, it is possible that as SD rats are healthy animals with normal/functional blood pressure-regulating mechanisms, the $\mathrm{HO}$ system may be exerting an effect in conjunction with other pathways for the homeostatic control of blood pressure. Therefore, the lessintense effect of the HO system would not alter other healthy pathways that exert an effect in concert with the $\mathrm{HO}$ system to regulate blood pressure. Whether this is an intrinsic homeostatic and/or defensive mechanism to maintain healthy conditions in SD rats within a certain physiological range remains unclear, and needs to be clarified in future studies. Importantly, the selectivity of the HO system in diseased conditions could be explored in the design of novel therapeutic agents.

In conclusion, our studies suggest that the suppression of the ET-1/ $\mathrm{PLC} / \mathrm{IP}_{3}$ pro-hypertensive axis and corresponding reduction in intracellular calcium, inflammation and oxidative insults may at least in part account for the blood pressure-lowering effects of heme-arginate. As UnX-DOCA hypertension is characterized by elevated ET-1, and ET- 1 stimulates PLC and $\mathrm{IP}_{3},{ }^{14}$ which in turn activate NF- $\kappa \mathrm{B},{ }^{15,16}$ the concomitant suppression of ET-1, PLC, $\mathrm{IP}_{3}, 8$-isoprostane and NF- $\mathrm{KB}$ by heme-arginate may be explored to attenuate the oxidative/inflammatory destruction of tissues. Collectively, our study unveils the effects of heme-arginate on PLC activity, and highlights the important link between the $\mathrm{HO}$ system, ET-1, the PLC-IP 3 signaling and NF- $\kappa \mathrm{B}$. More importantly, the regulation of NF- $\kappa \mathrm{B}$ by HO-1 may be important for cellular homeostasis, given the pleitropic effects of NF- $\kappa B$ signaling in many pathophysiological conditions, including inflammation ${ }^{44,45,48}$ and insulin resistance. ${ }^{49}$ Therefore, HO may be considered an important chaperon molecule not only for the regulation of blood pressure, but also for cellular defense and metabolic homeostasis.

Although the multifaceted interaction among these pathways is complex and needs further clarification, they seem to be more intimately connected. Thus, our findings would set the stage for future exploration of these pathways in the search for novel remedies.

\section{CONFLICT OF INTEREST}

The authors declare no conflict of interest.

\section{ACKNOWLEDGEMENTS}

This work was supported by the Heart and Stroke Foundation of Saskatchewan, Canada, and the Canadian Institutes of Health Research/University of Saskatchewan College of Medicine Bridge funding. We are grateful to James Talbot for technical assistance.
1 Lariviere R, Thibault G, Schiffrin EL. Increased endothelin-1 content in blood vessels of deoxycorticosterone acetate-salt hypertensive but not in spontaneously hypertensive rats. Hypertension 1993; 21: 294-300.

2 Pryor WA, Squadrito GL. The chemistry of peroxynitrite: a product from the reaction of nitric oxide with superoxide. Am J Physiol 1995; 268: L699-L722.

3 Fukunaga M, Yura T, Badr KF. Stimulatory effect of 8-Epi-PGF2 alpha, an F2isoprostane, on endothelin-1 release. J Cardiovasc Pharmacol 1995; 26(Suppl 3): S51-S52.

4 Fiore G, Florio P, Micheli L, Nencini C, Rossi M, Cerretani D, Ambrosini G, Giorgi G, Petraglia F. Endothelin-1 triggers placental oxidative stress pathways: putative role in preeclampsia. J Clin Endocrinol Metab 2005; 90: 4205-4210.

5 Khanna A, Simoni J, Wesson DE. Endothelin-induced increased aldosterone activity mediates augmented distal nephron acidification as a result of dietary protein. J Am Soc Nephrol 2005; 16: 1929-1935.

$6 \mathrm{Hu}$ CW, Webb RL, Jeng AY. Synergistic stimulation of aldosterone production in human adrenocortical carcinoma $\mathrm{NCl}-\mathrm{H} 295 \mathrm{R}$ cells by endothelin-1 and angiotensin II. J Cardiovasc Pharmacol 2004; 44(Suppl 1): S289-S292.

$7 \mathrm{Pu}$ Q, Neves MF, Virdis A, Touyz RM, Schiffrin EL. Endothelin antagonism on aldosterone-induced oxidative stress and vascular remodeling. Hypertension 2003; 42: 49-55

8 Blasi ER, Rocha R, Rudolph AE, Blomme EA, Polly ML, McMahon EG. Aldosterone/salt induces renal inflammation and fibrosis in hypertensive rats. Kidney Int 2003; 63: 1791-1800.

9 Artunc F, Amann K, Nasir O, Friedrich B, Sandulache D, Jahovic N, Risler T, Vallon V, Wulff P, Kuhl D, Lang F. Blunted DOCA/high salt induced albuminuria and renal tubulointerstitial damage in gene-targeted mice lacking SGK1. J Mol Med 2006; 84: 737-746.

10 Ortmann J, Amann K, Brandes RP, Kretzler M, Munter K, Parekh N, Traupe T, Lange M, Lattmann T, Barton M. Role of podocytes for reversal of glomerulosclerosis and proteinuria in the aging kidney after endothelin inhibition. Hypertension 2004; 44: 974-981.

11 Yan JC, Wu ZG, Kong XT, Zong RQ, Zhan LZ. Effect of CD40-CD40 ligand interaction on diacylglycerol-protein kinase $\mathrm{C}$ and inositol trisphosphate-Ca(2+) signal transduction pathway in human umbilical vein endothelial cells. Clin Chim Acta 2003; 337: 133-140.

12 Christ M, Meyer C, Sippel K, Wehling M. Rapid aldosterone signaling in vascular smooth muscle cells: involvement of phospholipase $C$, diacylglycerol and protein kinase C alpha. Biochem Biophys Res Commun 1995; 213: 123-129.

13 Andreis PG, Tortorella C, Malendowicz LK, Nussdorfer GG. Endothelins stimulate aldosterone secretion from dispersed rat adrenal zona glomerulosa cells, acting through ETB receptors coupled with the phospholipase C-dependent signaling pathway. Peptides 2001; 22: 117-122.

14 Zhang WM, Lin MJ, Sham JS. Endothelin-1 and IP3 induced Ca2+ sparks in pulmonary arterial smooth muscle cells. J Cardiovasc Pharmacol 2004; 44(Suppl 1): S121-S124.

15 Carter AB, Monick MM, Hunninghake GW. Lipopolysaccharide-induced NF-kappaB activation and cytokine release in human alveolar macrophages is PKC-independent and TK- and PC-PLC-dependent. Am J Respir Cell Mol Biol 1998; 18: 384-391.

16 Komarova SV, Pereverzev A, Shum JW, Sims SM, Dixon SJ. Convergent signaling by acidosis and receptor activator of NF-kappaB ligand (RANKL) on the calcium/calcineurin/NFAT pathway in osteoclasts. Proc Natl Acad Sci USA 2005; 102: 2643-2648.

17 Lavrovsky Y, Schwartzman ML, Levere RD, Kappas A, Abraham NG. Identification of binding sites for transcription factors NF-kappa B and AP-2 in the promoter region of the human heme oxygenase 1 gene. Proc Natl Acad Sci USA 1994; 91: 5987-5991.

18 Lavrovsky Y, Song CS, Chatterjee B, Roy AK. Age-dependent increase of heme oxygenase-1 gene expression in the liver mediated by NFkappaB. Mech Ageing Dev 2000; 114: 49-60.

19 Abraham NG, Kappas A. Pharmacological and clinical aspects of heme oxygenase. Pharmacol Rev 2008; 60: 79-127.

20 Horvath I, Donnelly LE, Kiss A, Paredi P, Kharitonov SA, Barnes PJ. Raised levels of exhaled carbon monoxide are associated with an increased expression of heme oxygenase-1 in airway macrophages in asthma: a new marker of oxidative stress. Thorax 1998; 53: 668-672.

21 Jeney V, Balla J, Yachie A, Varga Z, Vercellotti GM, Eaton JW, Balla G. Pro-oxidant and cytotoxic effects of circulating heme. Blood 2002; 100: 879-887.

22 Baranano DE, Rao M, Ferris CD, Snyder SH. Biliverdin reductase: a major physiologic cytoprotectant. Proc Natl Acad Sci USA 2002; 99: 16093-16098.

23 Stocker R, Glazer AN, Ames BN. Antioxidant activity of albumin-bound bilirubin. Proc Natl Acad Sci USA 1987; 84: 5918-5922.

24 Stocker R, Yamamoto Y, McDonagh AF, Glazer AN, Ames BN. Bilirubin is an antioxidant of possible physiological importance. Science 1987; 235: 1043-1046.

25 Balla G, Jacob HS, Balla J, Rosenberg M, Nath K, Apple F, Eaton JW, Vercellotti GM. Ferritin: a cytoprotective antioxidant strategem of endothelium. J Biol Chem 1992; 267: 18148-18153.

26 Hintze KJ, Theil EC. DNA and mRNA elements with complementary responses to hemin, antioxidant inducers, and iron control ferritin-L expression. Proc Natl Acad Sci USA 2005; 102: 15048-15052.

27 Jadhav A, Torlakovic E, Ndisang JF. Interaction among heme oxygenase, nuclear factor$\{$ kappa\}B, and transcription activating factors in cardiac hypertrophy in hypertension. Hypertension 2008; 52: 910-917.

28 Ndisang JF, Lane N, Jadhav A. Crosstalk between the heme oxygenase system, aldosterone, and phospholipase C in hypertension. J Hypertens 2008; 26 : 1188-1199 
29 Jadhav A, Torlakovic E, Ndisang JF. Hemin therapy attenuates kidney injury in deoxycorticosterone acetate-salt hypertensive rats. Am J Physiol Renal Physiol 2009; 296: F521-F534.

30 Ndisang JF, Zhao W, Wang R. Selective regulation of blood pressure by heme oxygenase-1 in hypertension. Hypertension 2002; 40: 315-321.

31 Jadhav A, Ndisang JF. Heme arginate suppresses cardiac lesions and hypertrophy in deoxycorticosterone acetate-salt hypertension. Exp Biol Med (Maywood) 2009; 234: 764-778.

32 Levere RD, Martasek P, Escalante B, Schwartzman ML, Abraham NG. Effect of heme arginate administration on blood pressure in spontaneously hypertensive rats. J Clin Invest 1990; 86: 213-219.

33 Reynolds LJ, Hughes LL, Yu L, Dennis EA. 1-Hexadecyl-2-arachidonoylthio-2-deoxy-snglycero-3-phosphorylcholine as a substrate for the microtiterplate assay of human cytosolic phospholipase A2. Anal Biochem 1994; 217: 25-32.

34 Ndisang JF, Gai P, Berni L, Mirabella C, Baronti R, Mannaioni PF, Masini E. Modulation of the immunological response of guinea pig mast cells by carbon monoxide. Immunopharmacology 1999; 43: 65-73.

35 Apak R, Guclu K, Ozyurek M, Karademir SE, Altun M. Total antioxidant capacity assay of human serum using copper(II)-neocuproine as chromogenic oxidant: the CUPRAC method. Free Radic Res 2005; 39: 949-961.

36 Clark JE, Naughton P, Shurey S, Green CJ, Johnson TR, Mann BE, Foresti R, Motterlini R. Cardioprotective actions by a water-soluble carbon monoxide-releasing molecule. Circ Res 2003; 93: e2-e8.

37 Bolognesi M, Sacerdoti D, Piva A, Di Pascoli M, Zampieri F, Quarta S, Motterlini R, Angeli P, Merkel C, Gatta A. Carbon monoxide-mediated activation of large-conductance calcium-activated potassium channels contributes to mesenteric vasodilatation in cirrhotic rats. J Pharmacol Exp Ther 2007; 321: 187-194.

38 Turkseven S, Kruger A, Mingone CJ, Kaminski P, Inaba M, Rodella LF, Ikehara S, Wolin MS, Abraham NG. Antioxidant mechanism of heme oxygenase-1 involves an increase in superoxide dismutase and catalase in experimental diabetes. Am J Physiol Heart Circ Physiol 2005; 289: H701-H707.

39 Purcell NH, Tang G, Yu C, Mercurio F, DiDonato JA, Lin A. Activation of NF-kappa B is required for hypertrophic growth of primary rat neonatal ventricular cardiomyocytes. Proc Natl Acad Sci USA 2001; 98: 6668-6673.
40 Wilson SH, Simari RD, Lerman A. The effect of endothelin-1 on nuclear factor kappa B in macrophages. Biochem Biophys Res Commun 2001; 286: 968-972.

41 Gukovskaya AS, Zinchenko VP, Petrunyaka VV, Khodorov BI, Evtodienko YV. Properties of different $\mathrm{Ca} 2+$ pools in permeabilized rat thymocytes. Eur J Biochem 1986; 161: 249-256.

42 Li X, Meng Y, Wu P, Zhang Z, Yang X. Angiotensin II and aldosterone stimulating NF-kappaB and AP-1 activation in hepatic fibrosis of rat. Regul Pept 2007; 138: 15-25.

43 Jadhav A, Torlakovic E, Ndisang JF. Hemin therapy attenuates kidney injury in deoxycorticosterone acetate-salt hypertensive rats. Am J Physiol Renal Physiol 2009; 296: F521-F534.

44 Sasaki T, Takahashi T, Maeshima K, Shimizu H, Toda Y, Morimatsu H, Takeuchi M, Yokoyama M, Akagi R, Morita K. Heme arginate pretreatment attenuates pulmonary NFkappaB and AP-1 activation induced by hemorrhagic shock via heme oxygenase-1 induction. Med Chem 2006; 2: 271-274.

45 Ndisang JF, Tabien HE, Wang R. Carbon monoxide and hypertension. J Hypertens 2004; 22: 1057-1074.

46 Gagov H, Kadinov B, Hristov K, Boev K, Itzev D, Bolton T, Duridanova D. Role of constitutively expressed heme oxygenase- 2 in the regulation of guinea pig coronary artery tone. Pflugers Arch 2003; 446: 412-421.

47 Hristov K, Altankova I, Gagov H, Bolton T, Boev KK, Duridanova D. Calcium-dependent changes in potassium currents in guinea-pig coronary artery smooth muscle cells after acute cobalt loading in vivo. Pflugers Arch 2004; 449: 16-25.

48 Yu P, Constien R, Dear N, Katan M, Hanke P, Bunney TD, Kunder S, QuintanillaMartinez L, Huffstadt U, Schroder A, Jones NP, Peters T, Fuchs H, de Angelis MH, Nehls M, Grosse J, Wabnitz P, Meyer TP, Yasuda K, Schiemann M, Schneider-Fresenius C, Jagla W, Russ A, Popp A, Josephs M, Marquardt A, Laufs J, Schmittwolf C, Wagner $\mathrm{H}$, Pfeffer K, Mudde GC. Autoimmunity and inflammation due to a gain-of-function mutation in phospholipase $\mathrm{C}$ gamma 2 that specifically increases external $\mathrm{Ca} 2+$ entry. Immunity 2005; 22: 451-465.

49 Chiang SH, Bazuine M, Lumeng CN, Geletka LM, Mowers J, White NM, Ma JT, Zhou J, Qi N, Westcott D, Delproposto JB, Blackwell TS, Yull FE, Saltiel AR. The protein kinase IKKepsilon regulates energy balance in obese mice. Cell 2009; 138: 961-975. 